\title{
Observation of the diffraction of a Helium-Neon laser beam on the monoatomic steps of a vicinal sapphire surface
}

\author{
Michel Lintz ${ }^{1}$ and Marie-Anne Bouchiat \\ Laboratoire Kastler-Brossel ${ }^{2}$ \\ 24 rue Lhomond \\ F 75231 Paris cedex 05
}

\begin{abstract}
"Vicinal" surfaces, with equally spaced monoatomic steps, can be obtained by thermally annealing single-crystals of materials like sapphire or silicon. We have observed the diffraction of a Helium-Neon laser beam on the monoatomic steps of a (0001) vicinal sapphire wafer surface. Diffraction angles were in the range $40^{\circ}$ to $50^{\circ}$, in agreement with the AFM-measured terrace length. Both sides of the wafer gave rise to lowest-order diffracted beams. This method allows one to determine surface characteristics such as the direction, average length and regularity of the terraces on the area illuminated by the laser. In the tested sample the direction of the crystal $\mathrm{C}$ axis could be shown to be uniform to within 5 arc seconds over a $12 \mathrm{~mm}$ area of the sample.
\end{abstract}

Keywords: Surface structure, morphology, roughness and topography; Light scattering; Vicinal single-crystal surfaces; Aluminium oxide

\footnotetext{
${ }^{1}$ Corresponding author. Fax: +(33) 1443234 34; E-mail: michel.lintz@oca.eu

${ }^{2}$ Affiliated to Ecole Normale Supérieure, Centre National de la Recherche Scientifique (UMR 8552), and Université Pierre et Marie Curie
} 
Sapphire $\left(\alpha-\mathrm{Al}_{2} \mathrm{O}_{3}\right.$ single crystal), and more particularly the (0001) surface is a widely used substrate for the epitaxial growth of metal or semiconductor thin films, or for silicon-on-sapphire technology. For many of these applications, the surface morphology can influence the quality of the semiconductor devices obtained. High temperature annealing of a sapphire surface is known to rearrange partly the originally disordered surface: it leads to a more or less perfect staircase of monoatomic steps, called a "vicinal surface" [1]. Steps at sapphire surfaces have been evidenced by reflection electron microscopy [2]. Later, monoatomic steps were studied in more detail using Scanning Probe Microscopies (SPM) like AFM (Atomic Force Microscopy) [3,4] or STM (Scanning Tunnelling Microscopy).

The resolution of SPM techniques allows atomic resolution imaging, and is invaluable: it has triggered the emergence of conceptually new fields in surface science. However, since the probe has to scan every point on the surface under scrutiny, these techniques are inherently slow. Even if the limited range (typically a few microns) of the piezo actuators used to translate the probe on the surface can be overcome at the expense of processing data corresponding to different scans, the maximum surface scanned is basically limited by the time required to scan the probe. Surface control of a full wafer is not compatible with the scanning probe microscopy throughput. Nor is the measurement of the roughness of a buried interface.

The very well-known technique of X-ray diffraction has reached a degree of sophistication which allows some surface parameters, including the terrace length, to be extracted from grazing-incidence X-ray diffraction measurements [5]. However, the corresponding diffracted intensities are very low, and experiments can only be performed using high quality X-ray sources available at synchrotrons. 
None of these techniques is a natural candidate for on-line inspection of large surfaces like wafers. Instead, one might prefer a holographic method using a coherent laser beam, in order to scan simultaneously the different points of a chosen area. Some difficulties can be foreseen:

- the light intensity diffracted from monoatomic structures is very small: for a Helium-Neon beam (wavelength $\lambda=633 \mathrm{~nm}$ ) diffracted on sapphire (index $n=1.8$ and steps of height $d=2.2 \AA)$, one obtains $[(d / \lambda) \times(n-1) /(n+1)]^{2} \approx 10^{-8}$

- while the use of visible light would offer the advantage and simplicity of visual observation, structures with typical transverse size smaller than the laser wavelength escape observation

- Light is scattered in all directions of space, which means that detection must be scanned in all directions, not unlike the AFM or STM probe.

However vicinal surfaces are a particular case, because light diffracted on the more or less regular grating of the monoatomic terraces is distributed around a given direction of space. Detection, either by a CCD camera or by visual observation, is made easier due to the limited solid angle and the correspondingly enhanced intensity. Moreover, the requirement of a laser wavelength shorter than the terrace length to be detected is less of a problem due to the increasing number of applications that require long terraces, of several hundred nanometers, and to the progress in laser diode technology. Commercially available Nichia blue laser diodes operate at $405 \mathrm{~nm}$, while even shorter wavelengths are available via frequency doubling.

In this letter we report on the observation of the diffraction of a $1 \mathrm{~mW}$ Helium-Neon laser beam from the monoatomic terraces of a vicinal sapphire (0001) surface, as a first step towards holographic mapping of a vicinal surface. 


\section{Experiment:}

We used $25 \mathrm{~mm}$ diameter (0001) sapphire wafers made by Kyocera ${ }^{3}$. These $0.5 \mathrm{~mm}$ thick wafers are superpolished on both sides (RMS roughness of a few Ångströms), with a parallelism of typically 100 microradian (for diagnosis of the parallelism, see for instance [6]). The miscut angle is specified by Kyocera not to exceed 10 arc minutes, but can be much smaller. Out of a batch of three wafers that were annealed at $1100^{\circ} \mathrm{C}$ for 1 hour, and then AFM tested, one was observed to have terraces of roughly $1 \mu \mathrm{m}$ length and $2.2 \AA$ step height (fig 1), which corresponds to a cut angle of approximately 1 arc minute, while the others had a terrace length shorter than $0.4 \mu \mathrm{m}$. The former wafer allowed observation of diffraction of a $633 \mathrm{~nm}$ laser beam at normal incidence, while the latter with short terrace length did not. Nor did wafers that had not been annealed. The He-Ne laser used ${ }^{4}$ had a excellent gaussian beam quality $\left(\mathrm{TEM}_{00}\right)^{5}$ with $0.6 \mathrm{~mm}$ diameter at $1 / \mathrm{e}^{2}$ intensity. Use of a frequency doubled $\mathrm{Nd}$ :YAG laser at $532 \mathrm{~nm}$ gave qualitatively similar results (except for the correspondingly smaller diffraction angle) but the beam quality was worse: stray light was difficult to reject, and the He-Ne laser was preferred.

Observation had to be performed in a clean room, otherwise, within minutes, ambient dust settling on the wafers soon gave rise to a light scattering level higher than the one due to diffraction on the monoatomic steps. The experimental set-up is extremely simple (fig. 2) and inexpensive. Apart from dust, the transmitted and reflected beams were the other potential source of significant stray light, and had to be completely absorbed. For this purpose we used beam dumps made of highly absorbing glass plates at Brewster incidence. As described in [8], the residual light level of these simple laser beam dumps can easily be made as low as $10^{-8}$ or $10^{-9}$.

\footnotetext{
${ }^{3}$ Contact: Marc.Lumbroso@kyocera.de; phone: (+33) 1451202 37;

http://www.kyocera.com/kicc/industrial/products/crystal.htm

${ }^{4}$ Melles Griot, reference 25 LHP 111. http://www.mellesgriot.com/pdf/0044.4-44.5.pdf

${ }^{5}$ For basics of laser physics, see for instance [7]
} 


\section{Basics of the diffraction by a grating}

We summarize here the basics of diffraction by a grating. More can be found in textbooks [9]; for a study of defects in gratings used for spectroscopy, see [10].

An optical surface covered by regularly spaced parallel grooves diffracts light in all directions perpendicular to the grooves, but the amplitudes diffracted by the different grooves add coherently only for some directions of space, for which the optical path difference is an integer multiple of the wavelength of light. For normal incidence of a collimated beam, the diffracted spots are found at angles $\theta_{d}$ such that

$$
\ell \sin \theta_{d}=k \lambda,
$$

where $\ell$ is the period of the grooves, $\lambda$ the wavelength of the light beam, and $k$ is an integer. If the period of the grating is not constant, a blurring of the diffraction angle $\theta_{d}$ is expected. If the direction of the grooves is not constant, a blurring in the azimuthal direction is also expected.

\section{Observations}

In a moderate darkness, visual observation of the beams diffracted at normal incidence on the vicinal surface was quite straightforward. The diffraction angle $\theta_{d}$ was typically $45^{\circ}$, in agreement with the formula $\lambda=\ell \sin \theta_{d}$ for first order $(k= \pm 1$ in eq. (1)) diffraction when $\ell \approx 1 \mu \mathrm{m}$ as seen from the AFM scan of fig. 1 , and $\lambda=633 \mathrm{~nm}$.

Diffraction at a surface gave rise to four diffracted beams: two $(k= \pm 1)$ beams forward and two $(k= \pm 1)$ backward, in agreement with the fact that, with the AFM-observed terrace length, only lowest-order diffraction could take place at $633 \mathrm{~nm}$. Sapphire being transparent, two sets of such diffraction patterns, each one originating at a given side of the wafer, are expected and were indeed observed. In fig. 2, the two diffracted angles have been chosen different for clarity. With our sample, they were often quite close (5 to $10^{\circ}$ difference). On the other hand, the two sets of diffracted beams had directions that 
differed by a rotation of roughly $30^{\circ}$ around the surface normal, indicating that the direction of the terraces differed by $30^{\circ}$ between the upper and lower side. Indeed diffraction was observed in directions perpendicular to the atomic steps seen on the AFM scans.

Diffraction patterns could be recorded in a Laue-type experiment: a photographic paper, with two holes to let the beam in and out, was rolled around the wafer in a cylindrical chamber. A diaphragm, added at the output of the HeNe laser, was needed to remove most of the fluorescence from the Helium-Neon laser discharge tube. With the 125 ISO photographic film used, a 5 minute exposure was found to be sufficient. On the unrolled film (fig. 3), the eight spots are visible. The four spots labelled "1" are rather ellipsoidal and originate from the same side. The four others, labelled "2", are sharper and more circular; they correspond to diffraction by the other side of the wafer.

Except for a limited region of the wafer, where only one set of four spots could be observed, the complete diffraction pattern was observed almost anywhere on the plate. This confirms that surface reconstruction took place on the whole surface of the plate, on both sides.

The point-to-point variation of the average diffraction angles indicates the variation of the terrace length (eq. (1)). On a $12 \mathrm{~mm}$ diameter area of the plate the average terrace length was found to be constant to about $\pm 10 \%$. Variations of the terrace lengths reveal variations of the cut angle and can originate either i) from an imperfect flatness of the plate, or ii) from a variation of the crystalline axes. If the crystalline lattice is assumed to be perfect, and using the $2.2 \AA$ value of the monoatomic step one concludes that surface flatness of each side of the plate is better than $\approx 60 \mathrm{~nm}$ on the considered area. The provider specifies a (short-scale) surface roughness of a few Ångströms, but not the flatness. Conversely, if one assumes a perfectly plane surface, then the crystalline axes at two different locations in this area differ by an angle not exceeding $\approx 2 \times 10^{-5}$ radian (roughly 5 arc seconds). Since 
it is unlikely that imperfections in the polishing would exactly compensate for imperfections in the crystal structure, these figures can be considered as upper limits. In the case of a thin crystalline sample, one way of discriminating between crystal structure and polishinginduced variations would be to look for correlated changes in the directions of the two diffracted beams, since it can be assumed that the crystal axes have the same direction on both sides of the thin sample.

The last quantity that can be monitored, at a given point on the sample, is the diffracted intensity distribution, namely its size, shape and structure. While the divergence of the incident laser beam was 1.35 mradian, diffracted spots were far more divergent, which suggests that, on the laser beam area, the period and direction of the steps are not strictly constant. For instance, the divergence of spots \#2 in fig. 3 was measured to be $\approx 5^{\circ}$. Thus the typical dispersion of the average terrace length on the laser spot can be estimated to be of $\approx \pm 4 \%$ of the average ${ }^{6}$. Finally, let us mention the structure of the diffraction spots. Fig. 4 shows a photographic recording of one diffraction spot: a 24×36mm camera without a lens was placed $145 \mathrm{~mm}$ away from the sample to record the intensity distribution. The typical size of the "speckle", $\approx 1.5 \times 10^{-3}$ radian corresponds to the diameter of the laser beam $\left(0.6 \mathrm{~mm}\right.$ at $1 / \mathrm{e}^{2}$ intensity) at the sample and contains no information about the crystal surface, but the intensity distribution is linked to the distribution of the steps at the surface of the crystal. Whether the actual distribution of the steps can be obtained from a digital recording of the diffracted intensity distribution is still an open question. In principle the answer is yes, at least if a holographic method is used, that is, by adding a reference beam in order to have both amplitude and phase information. This is an experiment we plan for the near future.

\footnotetext{
${ }^{6}$ Although the correct treatment would require Fourier transformation, an estimate can be made using eq. 1 , considering that if diffraction occurs at angle $\theta_{d}$, the corresponding period $\ell=\lambda / \sin \theta_{d}$ has to be present on the part of the grating which is illuminated by the laser.
} 
After several days in the clean room, some dust settled on the sample, and the surface had to be cleaned with spectroscopy grade acetone and Kodak cleaning paper. No effect on diffraction patterns was observed, at least qualitatively, after several such acetone cleanings. Later, acetone-resistant dust accumulated on the sample, giving rise to a prohibitive background of scattered light. Etching using 3:1 $\mathrm{H}_{3} \mathrm{PO}_{4}-\mathrm{H}_{2} \mathrm{SO}_{4}$ was decided (duration $10 \mathrm{mn}$, temperature $60^{\circ} \mathrm{C}$ ) to clean the sample. After rincing thoroughly with distilled water, diffraction patterns were still observable.

\section{Conclusion}

Diffraction of a $633 \mathrm{~nm}$ laser beam on the monoatomic steps of a vicinal (0001) sapphire surface has been observed, revealing the presence of relatively regular terraces on both sides of the wafer. Characteristics like direction, average length and regularity of the steps can be obtained with a very unsophisticated apparatus. Unlike scanning probe microscopies, this optical method allows large size wafers to be scanned in detail. Extension to magnesium oxide or silicon wafers is straightforward. These results are encouraging in the view of a holographic method, where the diffracted amplitude and phase would be recorded numerically and then processed to calculate the distribution of the steps at the surface.

Acknowledgments: We are grateful to M. G. Battuz and A. Hadj-Azzem (CRTBT, Grenoble) for the annealing of the substrates, and to V. Bouchiat, for the AFM scans of the samples, and for his help and interest in this work.

Appendices: (no appendix) 


\section{References}

[1] H. C. Jeong and E. D. Williams, Surf. Sci. Rep. 34(1999)171.

[2] Y. Kim and T. Hsu, Surf. Sci. 258 (1991) 131.

[3] M. Yoshimoto, T. Maeda, T. Ohnishi, H. Koinuma, O. Oshiyama, M. Shinohara, M. Kubo, R. Miura and A. Miyamoto, Appl. Phys. Lett. 67(1995)2615.

[4] J. R. Heffelfinger, M. W. Bench, C. B. Carter, Surf. Sci. 370(1997)L168.

[5] G. Renaud, Surf. Sci. Rep. 32(1998)1.

[6] E. Jahier, J. Guéna, Ph. Jacquier, M. Lintz, A. V. Papoyan and M. A. Bouchiat, Appl. Phys. B71 (2000) 561.

[7] O. Svelto, Principles of lasers, 4th ed. (Plenum Press, New York, 1998).

[8] M. Lintz. and A. V. Papoyan, Rev. Sci. Instr. 71 (2000) 4681.

[9] M. Born and E. Wolf, Pinciples of optics, 2nd ed. (Pergamon Press, Oxford, 1964) E. Hecht, A. Zajac, Optics (Addison-Wesley,1974)

[10] G. W. Stroke, in: Progress in Optics, Vol. 2, Ed. E. Wolf , (North Holland, Amsterdam, 1963) 


\section{Figures}

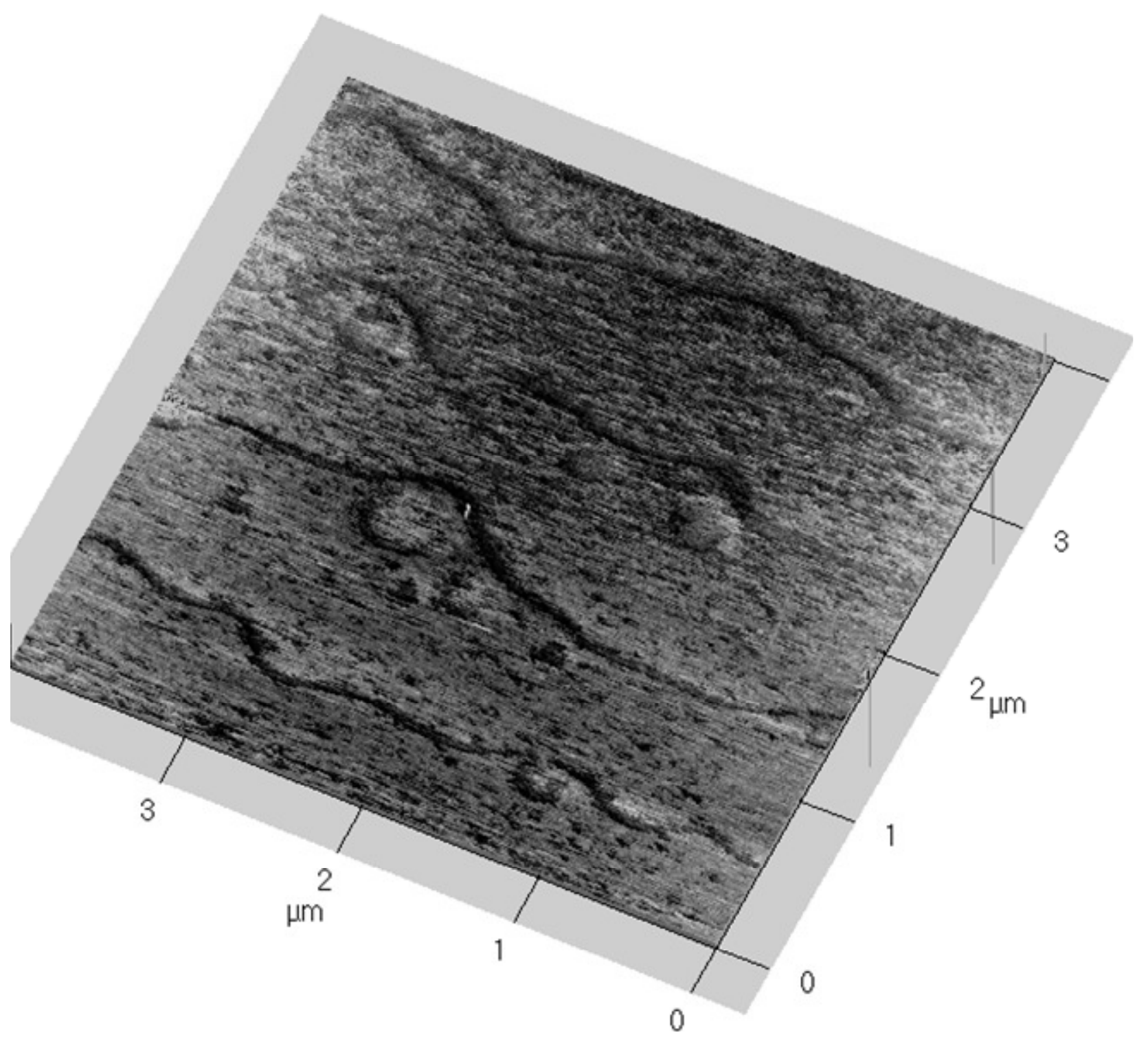

Fig 1: AFM scan of the surface of the annealed $\left(1100^{\circ} \mathrm{C}, 1\right.$ hour) sapphire wafer used in

the diffraction experiments. Surface scanned: $4 \mu \mathrm{m} \times 4 \mu \mathrm{m}$. The observed steps are $\approx 2.2 \AA$ high. 


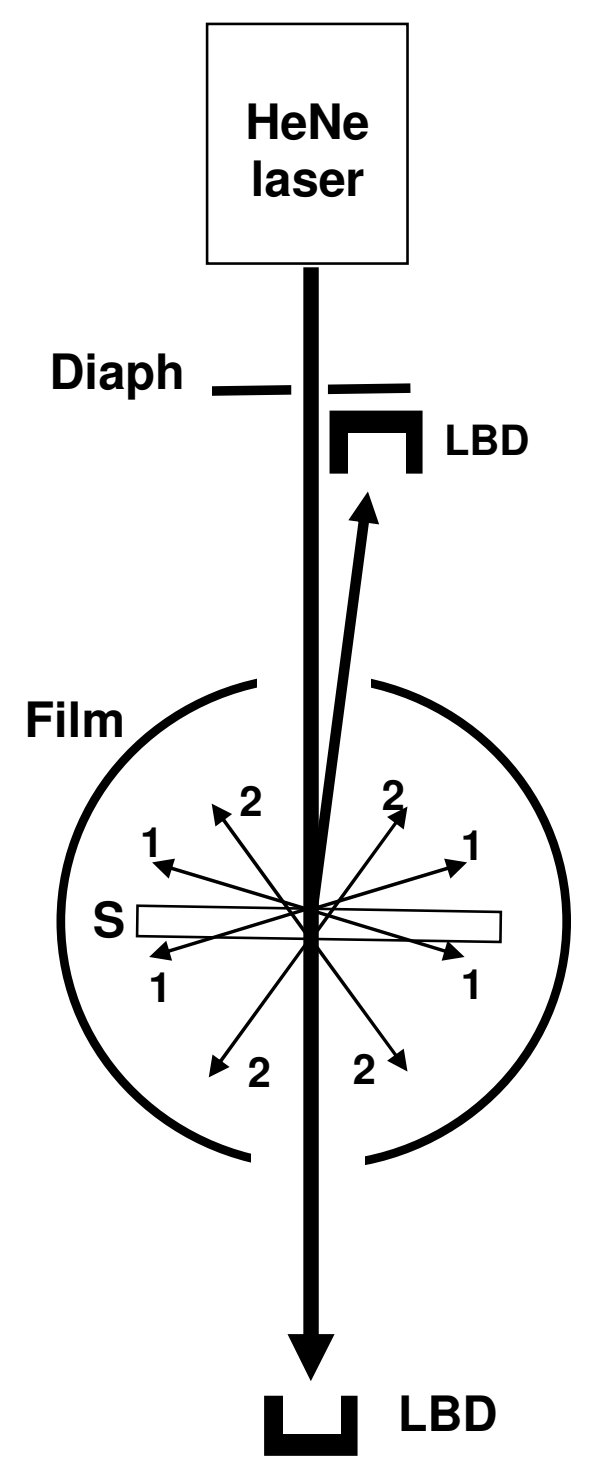

Fig 2: Experimental set-up. LBD: laser beam dump (described in ref. [8]). S: annealed sapphire sample. Film: photographic film inserted in a $\varnothing 30 \mathrm{~mm}$ cylindrical chamber, for photographic recordings of the diffraction pattern. Diaph: diaphragm, to reject uncollimated fluorescence from the Helium-Neon tube (necessary for photographic recordings). The four beams numbered "1" (resp. "2") are due to diffraction on the upper (resp. lower) side of the sample. 


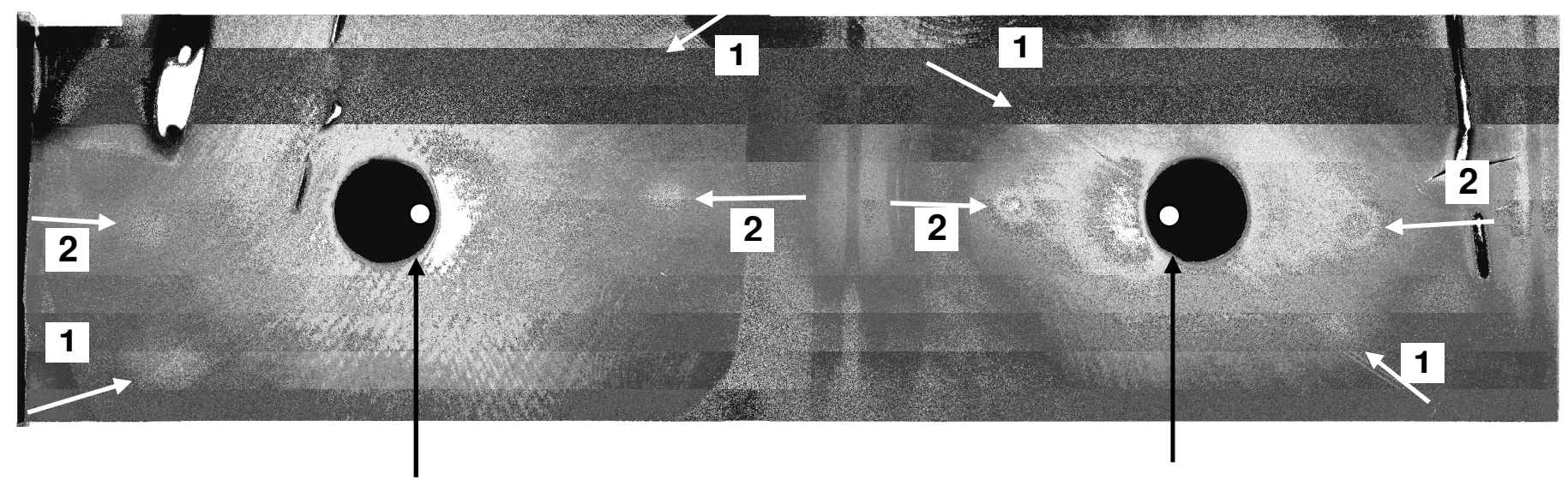

Fig 3: Typical "Laue-type" photographic recording of the diffraction pattern. The sample was not positioned exactly on the axis of the cylindrical chamber; this explains the imperfect up-down symmetry of the pattern. Exposure time: $5 \mathrm{mn}$. Film used: 125 ISO Ilford FP4 film. White dots: position of the laser beam at the input and output of the chamber. 


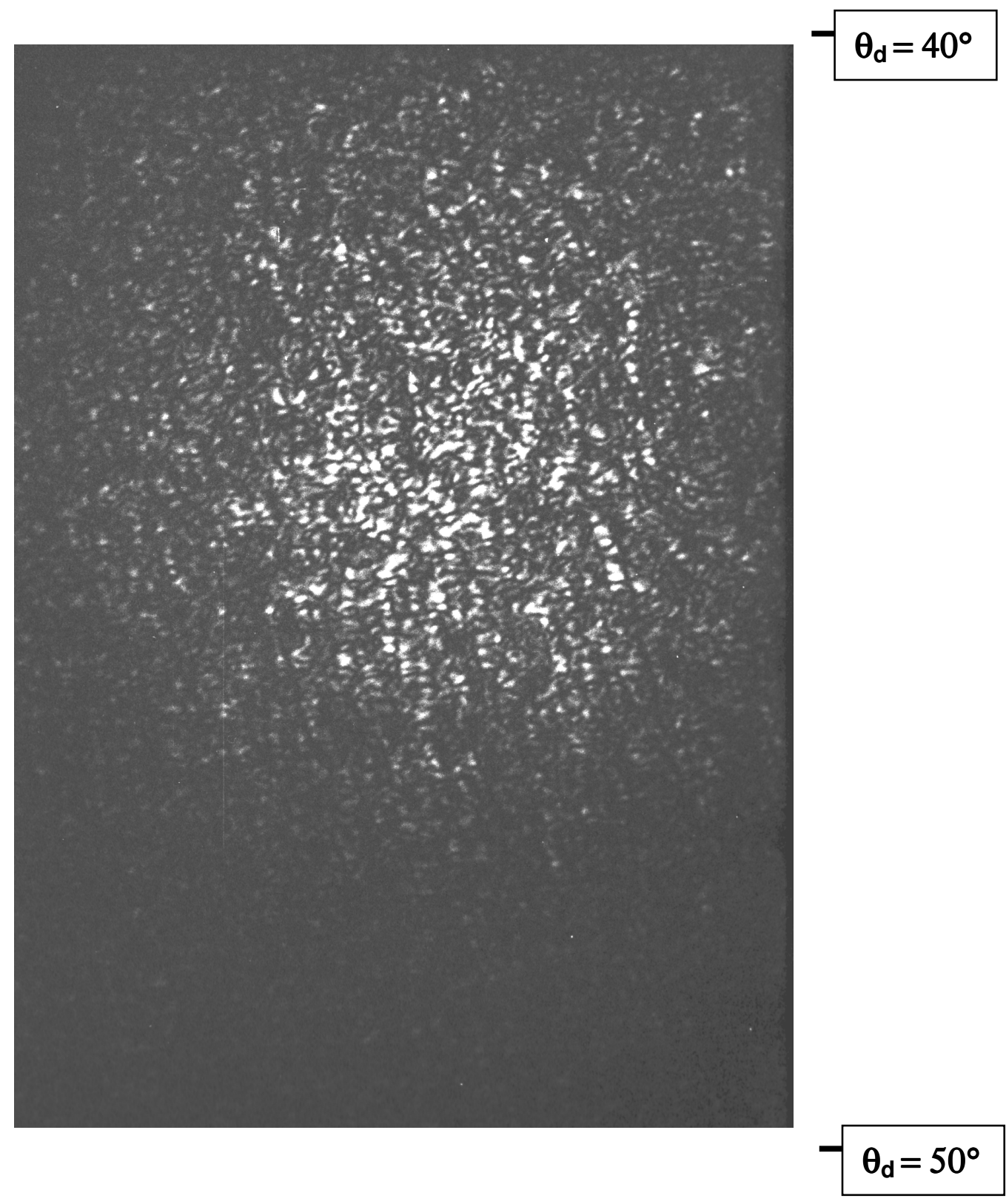

Fig 4: Photographic recording of the intensity distribution of a diffracted beam. The 400 ISO film was $145 \mathrm{~mm}$ away from the sample. Actual dimensions: $24.5 \mathrm{~mm} \times 19 \mathrm{~mm}$. Exposure time: $4 \mathrm{mn}$. The tested wafer area is not the same as in fig. 3. 